\title{
Natural antireflective microstructure of blue mussel shells and biomimetic replication
}

\author{
Ercai Pan* and Gangsheng Zhang \\ School of Resources, Environment, and Materials, Department of Materials Science and \\ Engineering, Guangxi University, Nanning, Guangxi 530004, China \\ *E-mail: ercaipan@163.com
}

Number of pages: 2

Number of figures: 1 

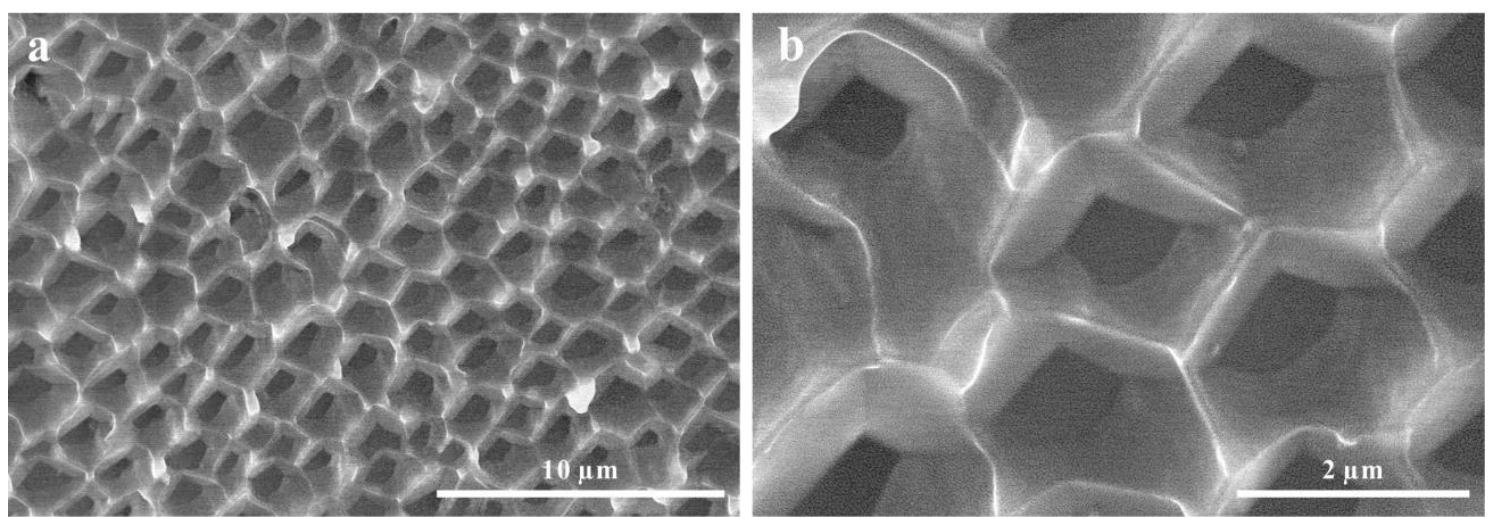

Figure S1. SEM images of the top views of the patterned PDMS film with inversed pyramidal microstructure. (b) is the amplification of (a).

Fabrication of patterned PDMS replica. Firstly, the liquid pre-polymer and curing agent were uniformly mixed and stirred at a weight ratio of 10:1 to synthesize the PDMS. Secondly, the pre-cured PDMS mixture was poured onto the treated shell and cured in a vacuum oven at $110{ }^{\circ} \mathrm{C}$ for 70 mins, which could obtain the highest hardness of the PDMS film. ${ }^{1}$ Finally, the PDMS was separated from the shell. (The PDMS [Sylgard 184 Silicon elastomer] was provided by Dow Corning Corporation of USA.)

\section{REFERENCE}

(1) Sun, J.; Wang, X.; Wu, J.; Jiang, C.; Shen, J.; Cooper, M. A. Zheng, X.; Liu, Y.; Yang, Z.;

Wu, D. Biomimetic Moth-eye Nanofabrication: Enhanced Antireflection with Superior Self-cleaning Characteristic. Sci. Rep. 2018, 8(1), 5438. 